\title{
Study on Recognition Method of Adhering Bars Based on Support Vector Machine
}

\author{
Guohua Liu *, Liangyu Li and Bingle Liu \\ School of Mechanical Engineering, Tianjin Polytechnic University, Tianjin \\ 300387, China \\ *Corresponding author:guohualiumail@163.com
}

\begin{abstract}
It is difficult to track, count and separate the moving bars at a high speed on production line for their overlap and accumulation. Therefore, it is necessary to establish a reliable, practical recognition and segmentation mechanism for the adhered bars. A new solution to the problem of bars adhesion is proposed: a support vector machine is constructed to recognize the adhesion type of bars by the feature vectors of training samples. The geometric feature values and moment feature values based on Blob regions in images are extracted, which is the input feature vector of support vector machine. The trained classifier is used for identifying the adhesion type of bars in images. Finally, classification and recognition is carried by support vector machine. The experimental results show that the recognition accuracy based RBF kernel achieves 100\%. The method is feasible and effective for the recognition and segmentation of the adhered bars.
\end{abstract}

Keywords: adhering bars; support vector machine; classification; recognition; bars

\section{Introduction}

With the popularization of negative deviation rolling technology, it is necessary to count and separate the steel bars moving at a high speed on production lines precisely to ensure the profit of the enterprises. The bars need to be reliably detected, located and determined the mutually positional relationship. Therefore, enterprises pay more and more attention to the method by which steel bars on production line can be quickly, accurately and automatically counted.

However, in practice, due to production process and complicated production environment of steel bars, severe noise usually exists in the acquired image for a couple of reasons: the light changing frequently, the motion blur of the image caused by the quick movement of steel bars, the dark or blue sections for oxidation. Especially, the image distortion caused by the monocular image acquisition will generate occlusion and adhesion phenomenon on the edge of images. In addition, misaligned bars will cause the same problem. All the problems will make it difficult to track, count and separate the steel bars on production line [1].

For all the adhesions of bars in the image, due to their same color and texture features, the adhering bars in the binary image will be in one connected domain after the image thresholding, they will be treated as one target. And this will cause erroneous results in subsequent processing. For the problem to the segmentation of adhering targets, many scholars have proposed different methods, such as mathematical morphology operations, improved watershed transform, and active contour tracking. The traditional watershed segmentation method is prone to result in over-segmentation, and the accuracy of object extraction is not high [2].

A recognition method based on neural network for bars on production lines after analyzing the geometry features of quasi-circular bars has been proposed in relief [3], and a template library is established. Experiment shows that this method has a long training 
time and a low recognition rate, furthermore, the segmentation result is poor [3]. Because corrosion and expansion method are irreversible, the method based on mathematical morphology often leads to inadequate and excessive segmentation for severely adhered bars.

So far, the problem still needs to be solved. In this paper, we will study on this issue in order to resolve the problem of adhering bars segmentation.First, the feature vectors are constructed with the extracted geometry features and moment features after image processing. Then the SVM method is used to identify the type of adhering bars. After this step, different optimal segmentation lines for different adhesion types can be constructed.

\section{Method}

Within the field of view, the moving bars on the production line may have these arrangements: (1) one single bar, (2) two adhering bars, (3) more than two adhering bars. For the bars image, each independent Blob region is identified after coarse segmentation, denoising and Blob analysis. However some of the target regions are composed of two or more bars, as shown in Figure 1. Each connected region may be different adhesions type. In order to ensure objective and accurate results of counting and separating, the type of adhering bars need to be identified and the adhering regions need to be divided into several independent single bars.

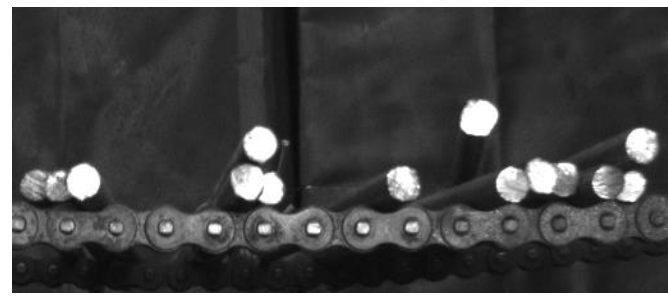

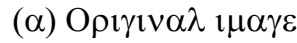

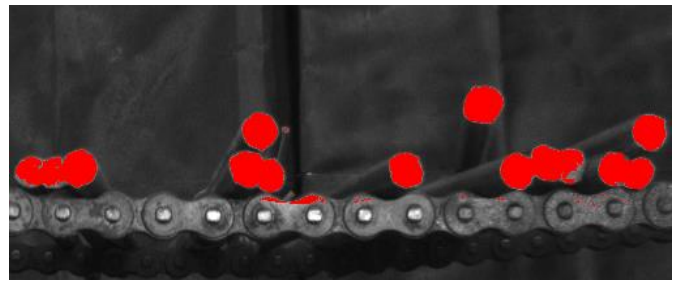

( $\beta) \mathrm{I} \mu \alpha \gamma \varepsilon \alpha \phi \tau \varepsilon \rho \tau \eta \rho \varepsilon \sigma \eta о \lambda \delta \imath \gamma \gamma$

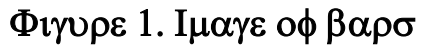

The classification for adhering bars moving on the production line is a complex multi-class discrimination issues. However, due to the restrictions of actual conditions, it is unrealistic to collect a large number of image samples for every adhering type. The support vector machine (SVM) as a new learning machine, was developed for the limited sample, it has better generalization ability compared with the traditional neural network method. Thus the support vector machine has its certain advantages for the classification of adhesion types, and it can also improve recognition accuracy.

After identify the type of adhering bars we could solve the segmentation problem, that is where and how to segment the connected domain. A segmentation algorithm for adhesions area is proposed in relief [4]:

(1) Analyze the concave convex features of adhesions region, detect possible segmentation points.

(2) Select viable segmentation route.

(3) Construct optimal segmentation line.

This region segmentation method using concave points searching mechanism has been widely used in cell adhesions and cereals segmentation, but it is complex and difficult to find the segmentation points [4].

In order to achieve better effect segmentation and counting, the type of adhering bars in the field of view must be determined first. If there are adhering bars, edges of the bar should be detected and smoothed, and concave points in this concave region should be detected according to the characteristics of each adhesions type. Then optimal segmentation line is constructed through a pair of concave points. In this way, the 
adhering bars can be separated from each other. The method of classification and segmentation can be seen in Figure 2.

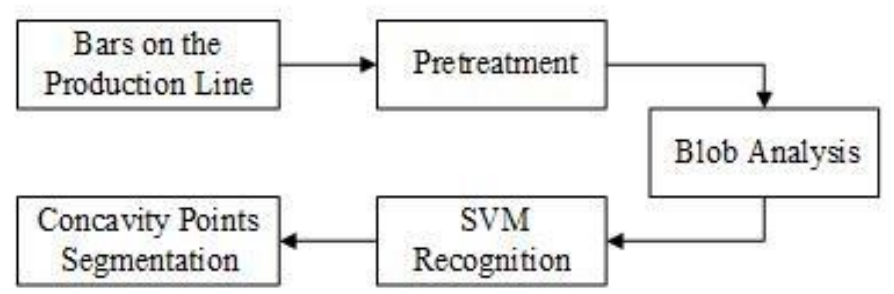

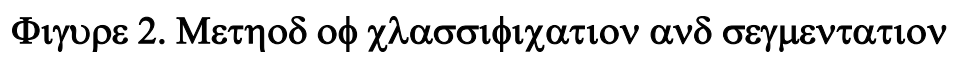

\section{Support Vector Machine}

Support vector machine (SVM) is a popular small sample learning method, which is based on the structural risk minimization principle and has a good performance for pattern classification problems. SVM was proposed by Vapnik, and it has many unique advantages. Compared with other learning algorithms, SVM appears to be a good candidate for several reasons: small sample learning, generalization ability, fast learning and evaluation for relevance feedback, flexibility.

For the realization of this algorithm, there is a nonlinear mapping; input vector is mapped to a high dimensional feature space, and optimal classification hyperplane is constructed in this feature space. From the formal point of view, SVM classification function is similar to neural network; the output is a linear combination of intermediate nodes, each intermediate node corresponds to a support vector, as shown in Figure 3.

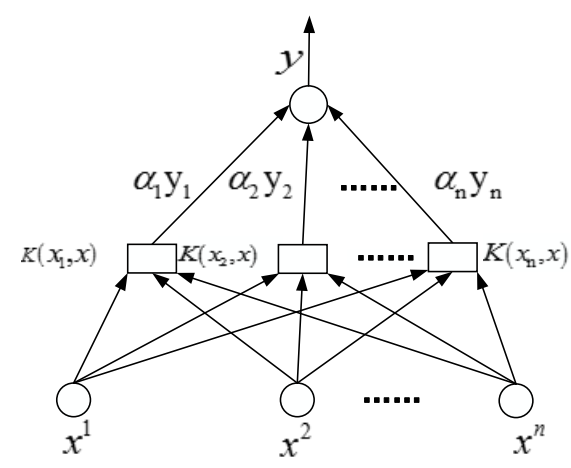

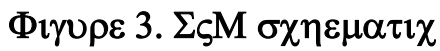

In the Figure 3, the output is: $y=\operatorname{sgn}\left\{\sum_{i=1}^{n} \alpha_{i} y_{i} K\left(x \cdot x_{i}\right)+b\right\}$, the weights is: $w_{i}=\alpha_{i} y_{i}$, $K\left(x \cdot x_{i}\right)$ is nonlinear transformation (inner product) of $x_{1}, x_{2}, \ldots, x_{s}$ based on $\mathrm{s}$ support vector, $x=\left(x^{1}, x^{2}, \ldots, x^{d}\right)$ is input vector.

According to the theory of functionals, as long as Mercer condition is satisfied, a kernel function $K\left(x_{i} \cdot x_{j}\right)$ can correspond to an inner product in a transform space. Therefore, in order to achieve linear classification after non-linear transform, appropriate inner product function $K\left(x_{i} \cdot x_{j}\right)$ is particularly important in the optimal hyperplane.

Under the premise of meeting Mercer conditions, different kernel functions correspond to different SVM algorithm. Among them, SVM kernel function is the key, it influences the accuracy of the classification results. Three types of kernel function are widely used currently. 
(1) Polynomial kernel function

$$
K\left(x, x_{i}\right)=\left[\left(x \cdot x_{i}\right)+1\right]^{\gamma}
$$

(2) Radial Basis Function

$$
K\left(x, x_{i}\right)=\exp \left\{-\frac{\left\|x-x_{i}\right\|^{2}}{g^{2}}\right\}
$$

(3) Sigmoid kernel function

$$
K\left(x, x_{i}\right)=\tanh \left[v\left(x \cdot x_{i}\right)+c\right]
$$

In the absence of prior knowledge guidance, results will be satisfactory by the use of radial basis function (RBF) [6-7].

\section{Experimental Analysis}

\subsection{Feature Extraction}

In this paper, images are obtained through the Basler plane array camera, whose resolution is $1296 \times 966$, frame rate is $30 \mathrm{fps}$, and collection field is $700 \mathrm{~mm} \times 200 \mathrm{~mm}$. One image can be acquired and processed within $30 \mathrm{~ms}$.

There is usually existing severe noise in the image obtained from the camera for a couple of reasons: shapes of bars sections are different; field environment is complex; light changes frequently. Therefore, image preprocessing is necessary in this paper, and the main means are image threshold segmentation and filtering [8].

The binary image is $B I(x, y)$ after pretreatment, and it can completely be divided into s sub-regions $B I_{i}(x, y)$, these sub-regions are formed by s-1 Blob regions (target regions) and a background [9].

The shape characteristic of the Blob region can be described by the moment [10], for one Blob image $\{I(x, y)\}$, its $(p+q)$ moment is as follows.

$$
M_{p, q}=\sum_{(x, y) \in I(x, y)} I(x, y) x^{p} y^{q}
$$

As shown in equation $(4),(x, y)$ is interior point or boundary point of Blob region, for the binary image, the value of $I(x, y)$ is 1 (Inside Blob) or 0 (Outside Blob). Moreover, moments sequences $\left\{M_{p . q}\right\}$ is only identified by $I(x, y)$.

Zero-order moment for the image can be described as equation (5).

$$
M_{0,0}=\sum_{(x, y) \in I(x, y)} I(x, y)
$$

Equation (5) is the area of Blob connected region.

The centroid of Blob region can be obtained by equation (6).

$$
\left(x_{c}, y_{c}\right)=\left(\frac{M_{1,0}}{M_{0,0}}, \frac{M_{0,1}}{M_{0,0}}\right)
$$

For some one Blob region, the roundness is showed as equation (7).

$$
\text { Roundness }=\text { major } / \min \text { or }
$$

In equation (7), major $=2 \sqrt{2} \sqrt{u_{c c}+u_{r r}+\sqrt{\left(u_{c c}-u_{r r}\right)^{2}+4 u_{r c}{ }^{2}}}$ is long axis for Blob region. $\min$ or $=2 \sqrt{2} \sqrt{u_{c c}+u_{r r}-\sqrt{\left(u_{c c}-u_{r r}\right)^{2}+4 u_{r c}^{2}}}$ is the short axis for Blob region. Among them, $u_{r r}=\left(\frac{1}{A}\right) \sum_{(r, c) \in R}\left(r-x_{c}\right)^{2}$ is Second-order row moment, $u_{c c}=\left(\frac{1}{A}\right) \sum_{(r, c) \in R}\left(c-y_{c}\right)^{2}$ is second-order column moment. $u_{r c}=\left(\frac{1}{A}\right) \sum_{(r, c) \in R}\left(r-x_{c}\right)\left(c-y_{c}\right)$ is second-order mixed moment, $A=M_{0,0}, \mathrm{R}$ is closed Blob region. 
When the bars are not adhered, the ratio of the Blob region area Area $_{B a r}$ and its convex hull area Area $_{\text {Convex }}$ is the largest, it is convexity, and it can be determined as a characteristic value to judge adhesions, the formula is equation (8).

$$
C 1=\frac{\text { Area }_{\text {Bar }}}{\text { Area }_{\text {Convex }}}
$$

When the height or the width of target Blob region exceeds the limit value, this region can be judged as adhesions. The external smallest rectangle parameters can be used as the ideal criteria. Among them $R(x, y)$ (width) and $R(x, y)$ (height) are the width and height of the rectangle $R(x, y)$.

Through the analysis of each Blob region, we can discover that external convex hull and perimeter of Blob region are different between adhesions and non-adhesions, which can be used as the basis for detecting the adhesions or not.

The compactness is:

$$
C 2=\frac{(\text { region_boder_length })^{2}}{\text { Area }_{\text {Convex }}}
$$

Through the analysis of geometric features and moment features of the bars under different conditions, area, roundness, convexity, width and height of external rectangle, compactness, perimeter are introduced as features to construct the feature vector in this paper.

\subsection{Sample Set}

Through the analysis of the bars production process, it is discovered that in addition to the vast majority of single bar, there may exist two bars adhesions, three bars adhesions (arranged in a line), three bars adhesions (stacked into a triangle). And other types are the combination of the foregoing typical types. Therefore, these four types are used to construct sample library. 141 samples are chosen, and 71 samples in the total samples are training samples and the other 70 samples are testing samples. In the training sample set, the number of single bar, two bars adhesions, three bars adhesions (arranged in a line) and three bars adhesions (stacked into a triangle) are respectively 19, 18, 18, 16. In the testing sample set, the number of single bar, two bars adhesions, three bars adhesions (arranged in a line) and three bars adhesions (stacked into a triangle) are respectively 18, 17, 17, 18. The image size is $247 \times 215$, and its grayscale is 256 .

In the training samples, the statistical results of 7 characteristic parameters for these four types can be seen in Figure 4.

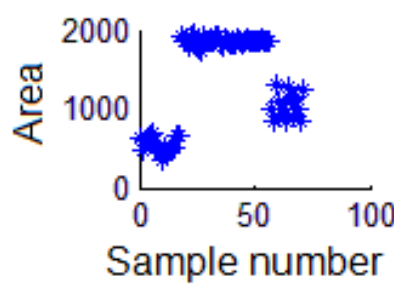

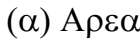

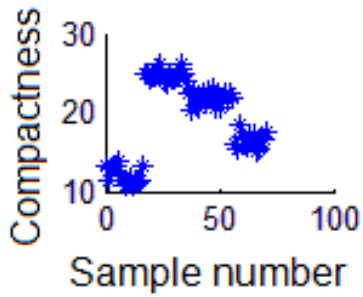

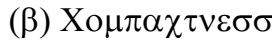




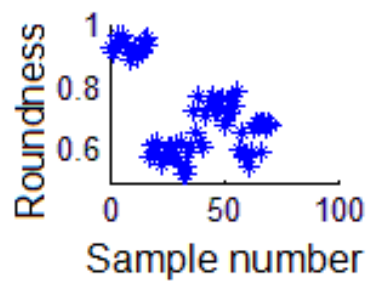

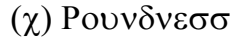

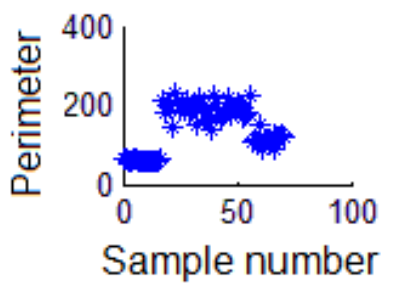

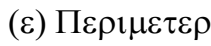

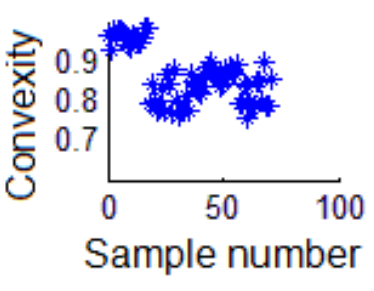

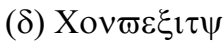

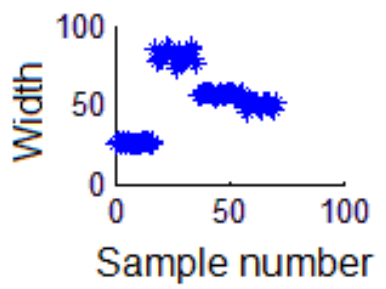

$(\phi) \Omega i \delta \tau \eta$

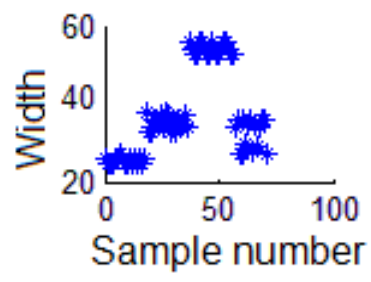

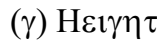

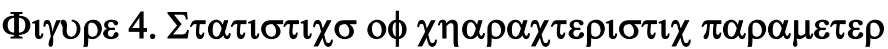

Greyscale map of characteristic parameters of 71 samples is obtained according to the periodic extension as shown in Figure 5.In the Figure 5, the abscissa axis represents characteristic parameters,different greyscale indicates different characteristic parameter,and the vertical axis shows 71 samples. The 4 classfication result based on 7 characteristic parameters easily can be seen in Figure 5.

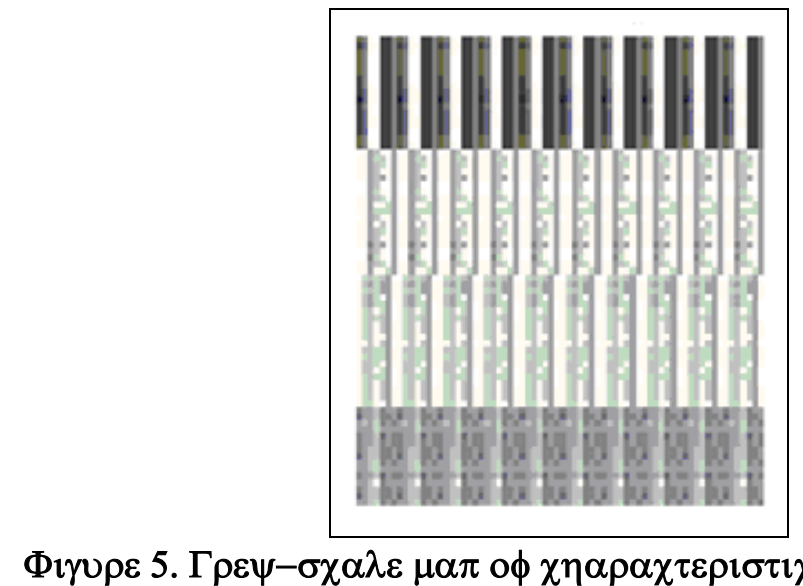

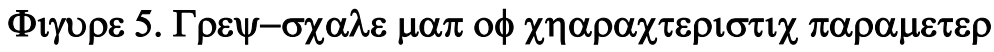




\subsection{Classification and Recognition Results}

All experiments in this paper are based on MATLAB; the SVM tool used in the experiments is Libsvm. Firstly, the image processing technology is used to optimize the acquired image and extract all kinds of characteristic values required for the experiment. Secondly, the training sample is used to train support vector machine and construct model. Finally, the trained model is used to detect the remaining test samples and get classification results.

From the above analysis, the selected radial basis function (RBF) will achieve better results, thus in this paper radial basis function is selected as the kernel function. The experiment shows that the kernel function affects significantly for training and testing results, the kernel function parameters are particularly important for the result, inappropriate parameters can lead to bad result. Therefore, the parameter optimization is very important for the experiment [11].

When the radial basis function (RBF) is determined, the classification results depend on its kernel function $\mathrm{g}$ and punishment factor $\mathrm{c}$. Therefore, these two parameters need to be optimized to get the best value. In this paper, cross-validation method is used to select the optimal parameters. The optimization result for support vector machine classifier (SVC) is shown in Figure 6. For logarithmic coordinate system in the Figure 6, c and g are respectively punitive parameters and kernel function width.

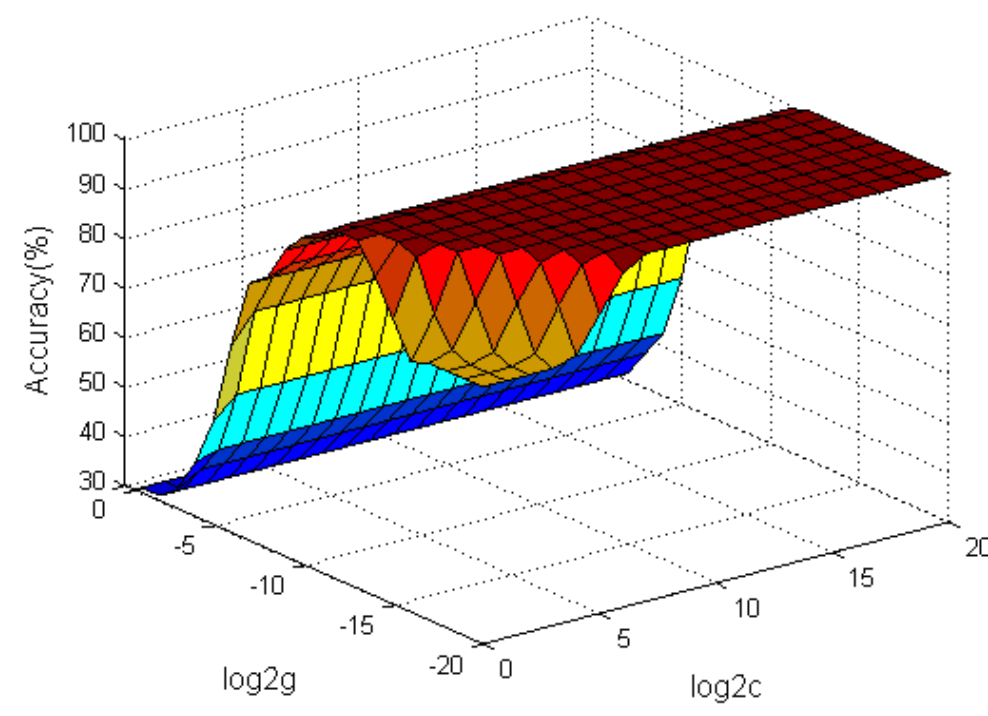

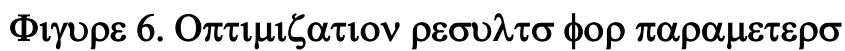

After getting optimized kernel function width $\mathrm{g}$ and punitive parameter $\mathrm{c}$, they are used to train training samples, and finally support vector machine model is obtained. Then the obtained model is used to test testing samples. The Figure 6 shows that the accuracy is $100 \%$ when $\mathrm{g}=1$ and $\mathrm{c}=0.00012207$.

The impact of different kernel functions for classification results are further analyzed in this paper, as shown in Table 1. As can be seen from the Table 1, radial basis function has the best performance.

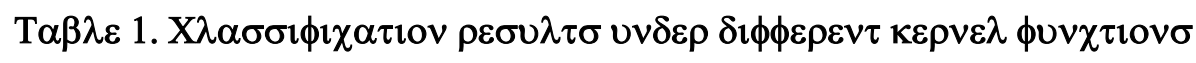

\begin{tabular}{|c|c|c|c|}
\hline $\begin{array}{c}\text { Kernel } \\
\text { functions }\end{array}$ & $\begin{array}{c}\text { Polynomial } \\
\text { kernel } \\
\text { functions }\end{array}$ & $\begin{array}{c}\text { Radial basis } \\
\text { function (RBF) }\end{array}$ & $\begin{array}{c}\text { Sigmoid kernel } \\
\text { functions }\end{array}$ \\
\hline Accuracy & $85 \%$ & $100 \%$ & $90 \%$ \\
\hline
\end{tabular}




\section{Conclusion}

For the identification problem of adhering bars on the production line, a new solution based on the support vector machine is proposed. The geometric feature values and moment feature values are the input feature vector of support vector machine. The trained classifier is used to identify the adhesion type of bars in image. The recognition accuracy based on this method achieves $100 \%$.

The experimental results show that, (1) The time consumption for this method to identify the adhesion type is $50 \mathrm{~ms}$, it can meet the requirements of real-time detection. (2) This algorithm is feasible and effective for the recognition and segmentation of the adhered bars.So it laid a solid foundation for improving the detection accuracy.

\section{References}

[1] D. Zhang, Z. Xie and J. S. Ai, "Research on rebar section positioning method in online rebar counting system", Chinese Journal of Scientific Instrument, vol. 31, no. 5, (2010), pp. 1173-1178.

[2] G. H. Wang, X. H. Zhou and L. L. Wei, "Image Segmentation Based on Watershed Algorithm", Computer Simulation, vol. 26, no. 5, (2009), pp. 255-258.

[3] Y. S. Zhang, Y. L. Fu, Z. G. Jiang and W. Z. Li, "Automatic Counting System of Steel Bars", Journal of Iron and Steel Research, vol. 18, no. 9, (2006), pp.1-4.

[4] S. Kumar, S. H. Ong, S. Ranganath, T. C. Ong and F. T. Chew, "A rule-based approach for robust clump splitting”, Pattern Recognition, vol. 39, no. 6, (2006), pp.1088-1098.

[5] N. Cristianini, J.Shawe-Taylor, "An Introduction to Support Vector Machines and Other Kernel-based Learning Methods", Cambridge University Press, (2000).

[6] X. X. Zheng, F. Qian, "Support Vector Machine with Gauss Kernel Classified Method and Model Selection", Computer Engineering and Applications, vol. 42, no.1, (2006), pp.77-79.

[7] J. Chorowskia, J. Wang and J. M. Zurada, "Review and performance comparison of SVM-and ELM-based classifiers", Neurocomputing, vol. 128, no. 27, (2014), pp. 507-516.

[8] M. Nixon, A. S. Aguado, "Feature Extraction \& Image Processing for Computer Vision", Academic Press Inc, London (2012).

[9] O.S. Ahmad, J. Debayle, N. Gherras, B. Presles, G. Févotte and J. Pinoli, "Recognizing overlapped particles during a crystallization process from in situ video images for measuring their size distributions", proceedings of the SPIE, (2011) June 28; Saint-Etienne, France.

[10] L. Ji, "Intelligent splitting in the chromosome domain", Pattern Recognition, vol. 22, no.5, (1989), pp.519-532.

[11] M. Sonka, V. Hlavac and R. Boyle, "Image Processing, Analysis, and Machine Vision", Thomson Learning and PT Press, Los Angeles (2003). 